\title{
Adapting as usual: integrative and segregative institutions shaping adaptation to climate change in local public administrations
}

\author{
MATTEO ROGGERO* \\ Resource Economics Group, Humboldt Universität zu Berlin, Berlin, Germany \\ ANDREAS THIEL** \\ International Agricultural Policy and Environmental Governance, University of Kassel, Kassel, Germany
}

\begin{abstract}
Local administrations play a key role in delivering adaptation to climate change. To do so, they need to address collective action. Based on transaction costs economics, this paper explores the role of so-called integrative and segregative institutions in the way local administrations adapt - whether their different functional branches respond to climate change collectively rather than independently. Through a comparative analysis of 19 climate-sensitive local administrations in Germany, the paper shows that variation in the way local administrations structure their internal coordination determines the way they approach climate adaptation. Under integrative institutions, local administrations adjust prior coordination structures to accommodate adaptation. Under segregative institutions, administrations move towards integrative institutions in order to adapt, provided they already 'feel' climate change.
\end{abstract}

\section{Introduction ${ }^{1}$}

Increasingly frequent extreme weather events and the waning prospects of a credible international commitment to curb greenhouse gases have given momentum to climate adaptation. Adapting to climate change consists in changing the ways individuals and society deal with those resources and assets threatened by climate impacts. However, it is not to be taken for granted that humanity will adapt to a future of changed climatic conditions: barriers impeding adaptation action in the face of expected climatic changes are many.

Part of what makes adaptation difficult is its character as collective action. Analyses of those incentives for or against collective action for adaptation haven't yet come about, though. As a result, we know surprisingly little about how the

\footnotetext{
*Email: matteo.mancini.roggero@gmail.com

**Email: thiel@uni-kassel.de

1 Supplementary material for this article is available at: https://goo.gl/DsDvbw.
} 
institutional structures shaping the provision of and access to climate-threatened resources are expected to perform under a different climate. More specifically, it's an open question whether and under what circumstances institutional change is to come about as a product of climate change, leading to adapted governance forms.

The present paper addresses that very question from the point of view of transaction costs economics, focusing on the way institutions handle interdependencies between functional units within a specific type of organization: local administrations. After framing the question in transaction costs economics terms, the paper presents a mid-N comparative analysis of 19 cases of adaptation by local administrations in Germany. Results show that institutional change aimed at delivering climate adaptation varies under different governance forms.

The paper is organized as follows: Section 2 reviews the relevant literature. Section 3 provides a framework for analysis. Section 4 details the materials used for empirical purposes and the methods employed to process them. Section 5 presents the results, which are discussed in section 6. Finally, section 7 offers some concluding remarks.

\section{Insights from the literature}

\section{Institutions, coordination and governance forms in climate adaptation}

Scholars of institutional economics are not necessarily familiar with climate adaptation. A short peek at the theoretical debates among adaptation scholars is therefore in order. Two elements of them are worth noting. First, adaptation scholars link adaptation barriers to collective action problems (Adger, 2003; Hinkel et al., 2010; Pahl-Wostl, 2009). Local administrations are key actors in the implementation of climate adaptation measures (Dodman and Satterthwaite, 2008; Few et al., 2011). Scholars question their capabilities in these respect, however (Amundsen et al., 2010; Keskitalo and Kulyasova, 2009; Næss et al., 2005).

The above helps in specifying the object of the present analysis. Leaving aside instances of individual climate adaptation such as double glazing in urban dwellings or livelihood diversification in subsistence agriculture communities, the present work addresses municipalities planning and executing adaptation measures in democratic market economies. A link to institutional theory is established by opening up the 'black box' of local administrations and exploring how actual governance forms shape collective action among their functional units. For that, transaction costs economics is employed (Coase, 1960; Williamson, 1998).

Transaction costs economics is an ideal candidate for the task ahead, as it addresses the link between variation in governance forms and variation in the attributes of the problems the same governance forms are meant to 
tackle. Originally focusing on the costs of using the market as an allocation system (Coase, 1960), transaction costs economics has proven insightful in many applications in natural resource management (Birner and Wittmer, 2004; Marshall, 2013; Roggero, 2015; Roggero and Fritsch, 2010; Thiel et al., 2012).

A starting point for the present analysis is Williamson's discriminating alignment hypothesis (Williamson, 1985), postulating that governance forms 'align' with the properties of the transactions they are to regulate. Transactions are the 'transfer(s) of goods and services across a technologically separable interface' (Williamson, 1985: 1). They are characterized by asset specificity, uncertainty and frequency. Based on these attributes, Williamson (1985) draws the line between market solutions and vertical integration: different institutional arrangements (markets, hierarchies or hybrid) perform differently in terms of the minimization of transaction costs based on the particular configuration of the three dimensions above.

Different characteristics will then be pertinent to different settings: many attempts have been made to provide suitable characterizations for different types of transactions (Birner and Wittmer, 2004; Hagedorn et al., 2002; McCann, 2013; Williamson, 1998), sometimes alternative to the ones above, sometimes complementing them (see Thiel et al., 2016 for an overview). Most relevant in our case is Hagedorn's focus on modularity and functional interdependence (Hagedorn, 2008; 2015) as discriminants between 'integrative' and 'segregative' institutions. While functional interdependence is a given among interdependent actors, the concept of modularity needs further articulation. This is done below.

\section{Modularity}

The modularity of transactions captures the decomposability of processes in selfcontained sub-processes (Baldwin 2007; Langlois 2002, 2006; Simon, 1962). Modularity has implications for the concept of transaction used by Williamson, which implicitly assumes that the activities at both ends of a transaction are bound together by a small, clearly defined set of interdependencies. In Baldwin's terminology, that is a 'thin crossing point' (Baldwin, 2007: 165). If activities are separated by a thin crossing point, the least-cost arrangement is to assign them to different units or 'modules'.

However, interdependencies between two activities may as well be many and complex, identifying a 'thick' crossing point. If that is the case, the leastcost arrangement is to have a single unit performing both activities. As a result, organizational boundaries will match 'thin' boundaries between activities, whereas 'thick' ones will fall within individual units. Transactions only take place across thin boundaries, whereas thick boundaries are dealt with through the internal organization of individual units. Figure 1 below provides an illustration.

The reader can easily visualize Figure 1 by referring to the case of a construction permit. Such a permit typically requires, on one hand, a set of environmental impact assessments (effects on the water table, drainage, risk of 
Figure 1. (Colour online) Modularity and organizational boundaries

Integration

Many interdependencies, Integrated units (no organizational boundaries)

Modularity

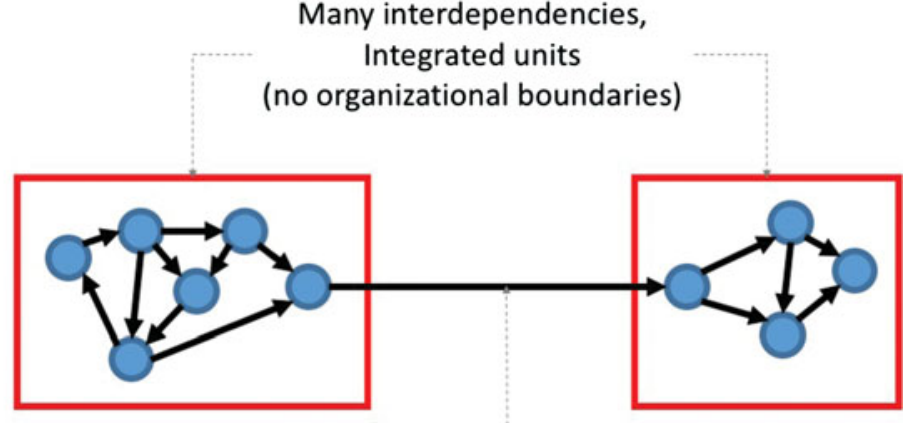

Few interdependencies, independent units

(separated by organizational boundaries)

Task

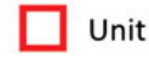

$\rightarrow$ Interdependency

contaminated soils being released, effects on flora and fauna, threats to endemic endangered species and the like). At the same time, it raises a series of planning issues (compliance with zoning regulations, building codes, labour and safety regulations, etc.). Environmental assessments are highly interdependent: they cannot make contrary assumptions concerning climate change, for instance. Similar considerations hold for planning issues.

Note however that environmental impact assessments and planning issues are only weakly interdependent with one another. Baldwin (2007) would thus see there 'thick crossing points' between the several sub-tasks making up both the environmental impact assessment and the planning issues. Between the environmental impact assessment as a whole and planning as a whole, she would see instead a 'thin' crossing point. She would then expect the two tasks to be accomplished by two separate units, divided by an organizational boundary at the thin crossing point.

This reasoning rests on an over-simplistic understanding of how organizations structure themselves, though. Organizational boundaries can be set for reasons other than minimizing the need for coordination, implying that highly interdependent activities may as well be assigned to different units. Yet transactions between highly interdependent activities can be modularized through rules such as technical standards (Baldwin, 2007): by shaping mutual expectations, rules greatly decrease the effort necessary to coordinate highly interdependent activities across different units of the same organization.

Bringing the above reasoning to its logical conclusions, organizational boundaries can be set everywhere, as long as rules can be established that modularize those functionally interdependent activities on both sides of the 
Figure 2. (Colour online) Integration and modularization

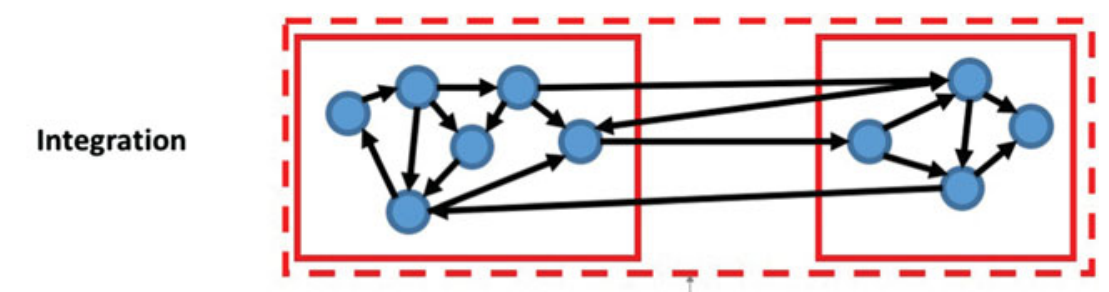

Integrative institutions (e.g.: working group)

Segregative institutions (e.g.: rules)

Modularization

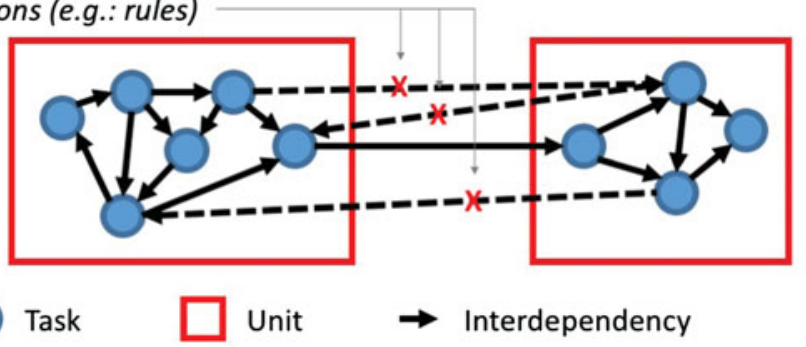

resulting transaction. As a result, organizational boundaries do not reflect much the degree of 'thickness' of the layer of interdependencies but rather whether such thick layers can be 'thinned' through rules that modularize the transaction between the actors on both sides of it. Figure 2 provides an illustration.

\section{Segregative and integrative institutions}

Against this background, the distinction between integrative and segregative institutions (Hagedorn 2008; 2015) can be put to use: rules can either segregate actors, allowing them to simplify things and neglect interdependencies (as in the case of rules modularizing the transactions introduced above), or integrate them, requiring them to coordinate action, embedding decisions in intense and direct social relations. To see the difference, compare institutions such as check-lists and meetings: the former allow actors within an organization to go ahead (or not) on a particular task on the basis of a narrow set of conditions. The latter require the same actors to meet and discuss various aspects of a task before acting on it.

This implies a qualitative shift from Williamson's initial concern - whether the same interdependent activities are best governed by a single integrated entity as opposed to plural ones. Here, a plurality of actors is assumed - the question being whether such actors act jointly (through integrative institutions) or independently (through segregative institutions). This, in turn, depends on the trade-off between the costs of integration and the costs of segregation (Figure 3).

Building up on the legacy of Buchanan and Tullock (1962), the basic logic of Figure 3 is that a decision-making process can internalize a varying amount 
Figure 3. Integration and segregation costs

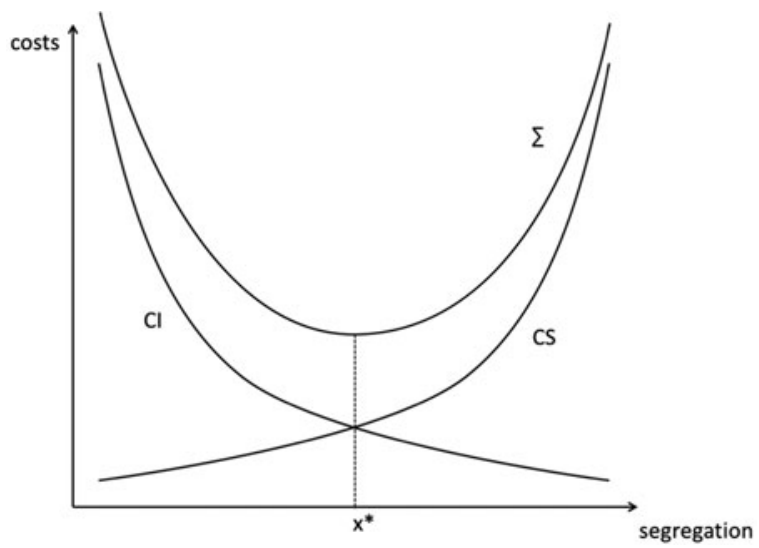

of interdependencies. The more interdependencies are internalized, the less costs are shifted upon third parties, lowering the amount of monitoring and sanctioning needed. Yet an increasing amount of interdependencies being taken into account, making decisions slower and more cumbersome. There is thus a trade-off between the costs of increased integration ('integration costs', or the costs of 'embedding decisions in social relations') and the costs of increased segregation ('segregation costs', or the consequences of deviating from a social optimum due to neglected interdependencies: see Hagedorn, 2015).

Both cost curves are conceptualized as convex, and thus non-linear. This characteristic is a legacy of both Buchanan and Tullock (1962) and Hagedorn (2015), and it seems reasonable to follow it: taking a third point of view into account while making a decision is certainly less exhausting (and hence marginally less costly) than taking the eleventh one into account, after balancing the first ten. Symmetrically, the more interdependencies are neglected, the more the biophysical system can be expected to approach its tipping point(s), showing resilience at first (the flat part of the segregation costs curve) and quickly transitioning to high costs (the steep part of the same curve).

Characterizing governance forms as a trade-off between different cost curves certainly offers an intriguing starting point for quantification exercises. Yet the quantification of transaction costs has so far proven challenging (McCann et al., 2005). It seems therefore safe to focus on the qualitative question here: whether the trade-off between integration and segregation costs will lean towards high segregation (that is: low integration) rather than towards low segregation (high integration), deeming the governance form segregative rather than integrative. That in turn depends on the relative shape of the integration and segregation costs curves, as shown in Figure 4.

Figure 4 portrays two situations with identical integration cost curves and different segregation cost ones. There's a qualitative difference between the two: 
Figure 4. Costs of integrative and segregative institutions
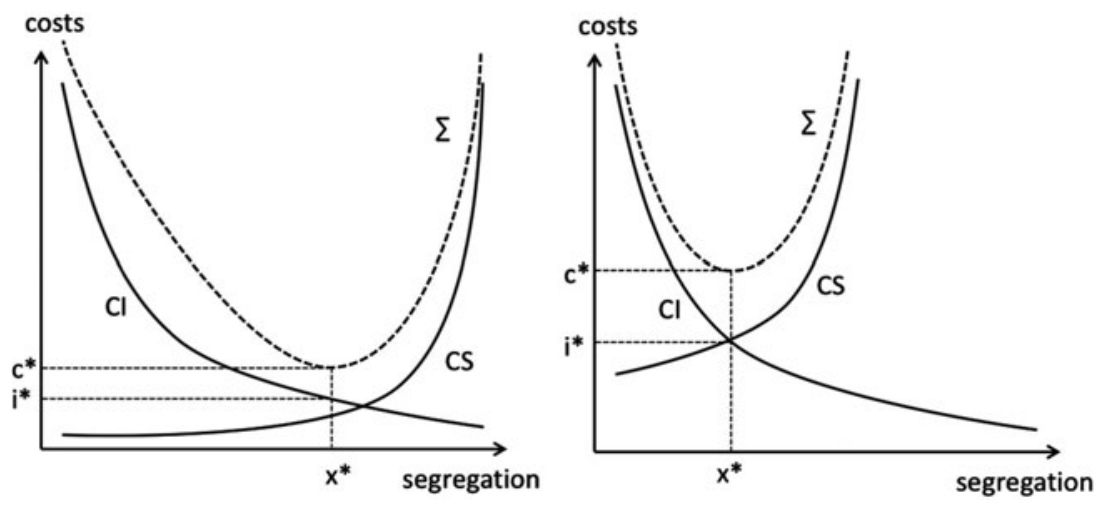

the diagram to the left describes a situation in which many interdependencies can be neglected before segregation costs escalate. The least-cost arrangement is one of low integration, governed by segregative institutions. In the right-hand diagram, segregation costs are higher, leading to integrative institutions.

\section{Towards an analytical framework}

\section{Setting the stage: the action situation}

Except for the very beginning of section 2, the concepts above have been articulated in general terms, without reference to the specific action situations at stake within local administrations. This becomes necessary now, since further specification will allow us to narrow down: (1) the nature of the interdependencies at stake; (2) the integrative versus segregative nature of the status quo institutions; and (3) whether and how climate change may trigger a shift towards segregative rather than integrative institutions. The last point represents this paper's research question.

Local admnistrations are conceptualized as comprising functionally diversified units, each one responsible for the direct provision of particular environmental resources from which citizens derive a benefit (recreation areas, air quality, trees, grass, etc.). Interdependencies among such units emerge from the biophysical links between such resources. Choices concerning the environment, for example, have direct effects on allergies: as a result, allergy plans may be completely off-set by choices concerning parks and alleys. Health units (designing allergy plans) therefore need to work closely with environmental units (designing parks and alleys). Failing to do so may lead to significant administrative and political transaction costs, exposing individual units and the administration as a whole to various kinds of pressures, from open critique to litigation in court.

Administrations vary in terms of their internal coordination. Two elements are particularly crucial: (1) the availability of coordination structures, either formal 
or informal; (2) the role of ad-hoc, informal versus more structured coordination. We interpret diversity in these regards as the result of varying integration costs. At the lower, less 'integrated' end of the spectrum, administrations address interdependencies in an ad hoc way, while at the higher, more 'integrated' end, they coordinate in a structured fashion, be it through informal but regular exchanges or by entrusting particular units to facilitate coordination.

Finally, climate change acts as a driver of change by affecting the resources at stake, changing those baseline conditions on which local administrations base their work: climate change introduces uncertainty concerning the way the ecosystem at stake will perform, potentially pushing some of its features outside the boundaries for which knowledge exists. Changing vegetation periods, for example, shift the temporal and spatial distribution of pollen release, potentially offsetting schemes that minimize discomfort to the allergic, as medications may be made available too late or too early - the more so if the environment department adapts to climate change by choosing new plants and tree species without consulting the health department first.

\section{Implications for the cost curves}

Integration cost curves link integration costs to actual integration efforts, raising the question whether public administrations and their individual units face different cost curves. To the contrary, it is safe to assume that they all face the same cost curve and find themselves at different points along it. This may seem a strong assumption. Yet administrations have a rather narrow set of options for involving stakeholders in decision making, making it unlikely that decisionmaking processes of similar complexity lead to significantly different decisionmaking efforts across administrations. By the same token, larger decision-making efforts imply a larger number of interdependencies being taken into account.

Whereas integration cost curves are the same for all administrations, segregation cost curves are not. They depend on perceived local biophysical circumstances, causing the physical attributes of the 'transaction' at stake to vary across administrations. Specifically, administrations may differ in terms of how they subjectively perceive the nexus between the interdependencies they can neglect and the transaction costs they can expect to face if they implement inadequate or unpopular solutions.

If segregation cost curves differ across units, but integration cost curves do not, a link can be established between the investments in coordination observed and the subjective perception of the biophysical conditions justifying such investments. Observing integrative institutions denotes high investments in coordination, which in turn reveal perceptions of a complex system of highly interdependent environmental resources. Observing segregative institutions, instead, implies resources being conceived of as simple and loosely interconnected, making it worthwhile to keep integration costs low. With reference to Figure 4, observing high rather than low $i *$ (and the corresponding 
$x *$ ) indicates whether the segregation curve at stake is similar to the one in the left-hand diagram rather than the one to the right, respectively indicating segregative versus integrative institutions.

\section{The effects of a changing climate upon cost curves}

The discussion above distinguishes integrative and segregative institutions by relying on integration and segregation cost curves. Let us now address the effects of a changing climate upon such curves, and the institutional change potentially ensuing. This has pointed to an increase in uncertainty, raising the question how it is expected to affect integration and segregation curves.

Integration cost curves express the effort necessary in order to address a certain number of interdependencies in a given decision. Additional uncertainty doesn't change how complex it is to address any number of interdependencies per se. Rather than that, it may require more interdependencies to be taken into account. This implies movements along the curve rather than a shifting of the curve itself. To the contrary, increased uncertainty is likely to affect segregation cost curves. Increased uncertainty makes the same amount of interdependencies costlier to deal with in light of the potentially larger number of unintended effects to be taken into account. This equals to an 'upward shift' of the segregation costs curve, causing a shift of the least-cost arrangement towards more integration. ${ }^{2}$

This reasoning effectively provides a transaction costs explanation for the popularity of collective action in the literature on local adaptation. The relevant question, though, is whether the upward shift will be different under segregative and integrative institutions. Recalling that the distinction between integrative and segregative governance forms reflects different segregation cost curves (see Figure 4), this question becomes one of disentangling the interplay between the shape of the segregation cost curve and the upward shift caused by increased uncertainty.

Under integrative institutions, any further upward shift of the segregation cost curve can do nothing other than call for further integration. Additional uncertainty, in other words, won't trigger a change from integrative to segregative institutions. Furthermore, the segregation cost curve meets the integration cost curve at a very high slope - close to the infinitely costly situation of full integration. Because of that, enormous upward shifts are necessary for substantial increases in integration. More likely, the upward shift will only trigger a moderate increase in integration.

More interesting is the case of segregation cost curves leading to segregative governance arrangements. These represent situations characterized by comparatively few well-understood interdependencies, which can be either left out of decisions or addressed through rules without causing substantial

2 The interested reader can find a diagrammatic illustration of this in the supplementary material see Figure X1. 
implementation costs. In the face of increased uncertainty, this situation may give rise to two alternative scenarios: (1) that interdependencies are so well understood that uncertainty hardly affects implementation costs; (2) that uncertainty particularly affects those interdependencies previously considered well understood.

In the first scenario, the segregation cost curve retains its main characteristic: that implementation costs become substantial only very late. Without a qualitative change in the segregation cost curve, no qualitative change from segregative to integrative institutions is expected. In the second scenario, however, the segregation cost curve implies implementation costs that increase rather early, becoming similar to the case of integrative institutions. Increased uncertainty therefore results in a shift towards integrative institutions. ${ }^{3}$

\section{Materials and methods}

\section{Data collection: case selection and coding}

The empirical material for this paper was collected within the econCCadapt project between 2012 and 2015. During econCCadapt, the authors of this paper carried out an institutional analysis of local climate adaptation, focusing on municipalities along the Lower Rhine and the North Sea coast. Deliverables are available on the project's website that describe in detail the data we employ here. For the reader's convenience, a brief summary is provided below.

The project focused on heat, heavy rains and floods in 'climate-sensitive' local administrations. These were identified through indicators by the German Ministry of Transport, Construction and Urban Development (Gruehn et al., 2010). In short, local administrations on the Lower Rhine or on the North Sea coast, including the 'city-states' of Hamburg and Bremen, were identified as 'highly sensitive' to climate change if they featured in the highest quartile for built surfaces and at least one more sensitivity indicator (elderly citizens for heat; infrastructure for heavy rains and flood risk areas for floods).

This led to 25 local administrations, six of which dropped out of the study for different reasons (representatives not available, unreachable, or 'special' and thus not comparable administrative constructs, etc.). In the remaining 19, semistructured interviews were carried out with representatives of the environment, planning, environment and water departments. For each local administration, interview materials were consolidated into a case study narrative, which was then sent back to the interview partners for comments, additions and revisions.

The revised case study narratives were subsequently coded along a number of variables capturing, on one hand, the way 'horizontal' coordination across units and departments comes about and, on the other hand, the way climate impacts are perceived and translated into adaptation processes. This has

3 Both scenarios are illustrated in Figure X2 in the supplementary material. 
led to a table containing 19 records of 22 different descriptors. ${ }^{4}$ So much information allows for a much broader range of analyses than of interest here. Let us therefore focus on those descriptors we will rely upon for testing the hypotheses above: 'Coord_Units', 'Early_Coord', 'Vulnerability_ACTUAL', 'Need2Adapt_Projected', 'Fine_Tuning', 'Ongoing_Process'.

The interested reader can refer to the supplementary material, ${ }^{5}$ where Table X1 reports in tabular form how each municipality scores against the above-mentioned descriptors - cell values expressing whether the given descriptor is observed or not. The case studies were originally coded using fuzzy-set membership scores. For our purposes, scores were converted into binary expressions, allowing for more intuitive graphical representations (Venn diagrams or, in this case, two-by-two matrices rather than scatterplots).

Let us now turn to the meaning of the individual descriptors. The first two reflect the municipalities' diversity in terms of organizational forms. 'Coord_Units', in particular, expresses which municipalities have units with coordination purposes. These are usually individual positions and/or task forces addressing a specific issue (typically: social integration, sustainability, housing), and doing so by coordinating the action of other units rather than acting themselves.

'Early_Coord' captures instead informal coordination practices and expresses whether these are dominant over formal procedures: in those local administrations, officials first coordinate informally and then formalize their decisions through the regular procedures. Please note that both descriptors refer to administrative action in general and not in relation to climate adaptation. The analysis would otherwise become tautological.

The remaining descriptors provide a picture of how the municipalities perceive the adaptation ahead: whether municipalities already 'feel' climate change ('Vulnerability_ACTUAL'); whether they perceive the need to adapt in the future because of this ('Need2Adapt_Projected'); whether their current coordination structures are capable of addressing climate change by some minor adjustments ('Fine_Tuning'); and whether actual adaptation processes are currently underway ('Ongoing_Process').

\section{Data processing: set relations}

In order to test whether the available evidence supports our theoretical expectations, the present papers employs set-theoretic methods as laid down by Ragin (2006) and Schneider and Wagemann (2012). It relies on the statistical package by Quaranta and Schneider (2013). Set-theoretical methods focus on exploring set relations. The aforementioned descriptors represent therefore 'sets',

4 See supplementary material, Figure X3.

5 See note 1. 
such as the 'set of all municipal administrations featuring coordination units' ('Coord_Units').

For each descriptor, values of one and zero respectively indicate the membership and non-membership of the given municipality in the corresponding set. Once the membership of the cases under study in the sets of interest is clear, the analyst can turn to exploring 'set relations', or relations between sets: whether, for example, the set of municipalities featuring coordination units ('Coord_Units') is a subset, a superset or is identical to the set of municipalities featuring ongoing adaptation processes ('Ongoing_Process'). This is done by assessing the distribution of the available cases across the intersections of the two sets at stake: how many cases are observed belong to both sets, to none, or to either one of them but not the other.

Once set relations are established, using them as a tool for (social) scientific inquiry is done by interpreting them as sufficiency and/or necessity claims: if 'Coord_Units' is a subset of 'Ongoing_Process', then having coordination units is a sufficient condition for having adaptation processes underway. Symmetrically, if 'Early_Coord' is a superset of 'Fine_Tuning', then informal coordination efforts prior to formal, official ones are a necessary condition for coordination structures to handle adaptation via minor, incremental adjustments.

In practice, the above translates into simple two-by-two matrices, coupled with a small set of 'measures of fit' expressing the relative frequency of cases compliant with versus contradicting a particular claim. Matters of space preclude an exposition of such measures, their meaning, interpretation and pitfalls. In the following, we will therefore report them for matters of completeness but will not rely on them for explanatory and illustrative purposes: readers will only need to keep in mind that we will not strive for full consistency but rather accept, within reason, a minimal amount of contradictory evidence. The interested reader can refer to Schneider and Wagemann (2012) for a detailed treatment.

\section{Analysis and results}

The present section explores empirically the question whether administrations characterized by segregative rather than integrative institutions react differently to the increased coordination required to address the uncertainty brought about by climate change. It does so in a stepwise fashion, first distinguishing local administrations based on their reliance on integrative versus segregative institutions (Analysis 0), subsequently addressing integrative institutions (Analysis 1), and finally focusing on the specifics of segregative institutions (Analysis 2). All analyses are based on the set relations illustrated in Figure 5.

\section{Analysis 0: Integrative versus segregative institutions in local administrations}

Local administrations can embed decisions in social relations both in formal terms and through informal practices - in ways that are not mutually exclusive. 
Figure 5. Set relations

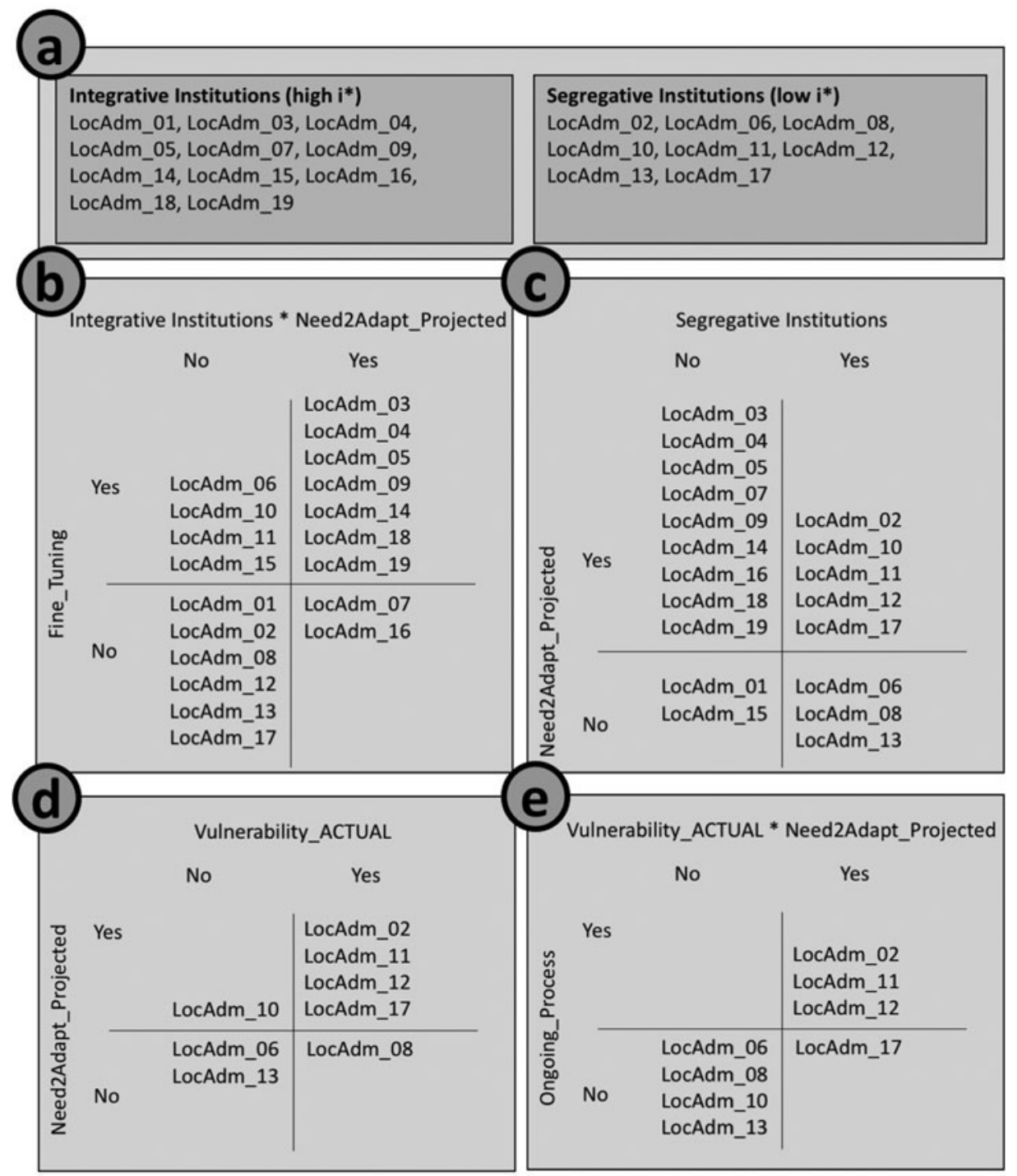

The available dataset provides two descriptors respectively capturing the former and the latter: 'Coord_Units' and 'Early_Coord'. Local administrations presenting both are certainly investing substantial manpower in coordination activities (high ' $i$ '' in Figure 4, right-hand diagram) and will thus be considered as characterized by integrative institutions.

All other local administrations will instead count as segregative: either they do not invest manpower in coordination ('Coord_Units' not observed), or they rely on procedures rather than on social interaction ('Early_Coord' not observed), 
or both. Figure $5 \mathrm{a}$ shows the resulting distribution of local administrations between the two sets, showing a rather balanced split. More importantly, though, some anecdotal evidence from the case studies will help illustrate the qualitative difference between the two sets ('Coord_Units' and 'Early_Coord'). Let us first address LocAdm_10 and LocAdm_11, where early coordination and coordination units respectively are observed, but not together.

LocAdm_10 has some cross-cutting units, but they are not representative of the way things are done there. Early coordination does take place, but only if the complexity of the issues at stake justifies it. The organization of LocAdm_11 foresees instead positions in coordination roles; it prefers however formal over informal coordination processes, so that different standpoints are brought forward independently of one another and are integrated at a later stage. The qualitative picture emerging from the case studies is thus one where municipalities do feature some degree of integration, but impose limits on it.

In comparison, the question for LocAdm_09 (featuring both early coordination and coordination units) is rather one of choosing the right integrative institution among many. The rationale emerging from the interviews is that some issues are best dealt with through formal integrative institutions, other ones through informal processes. Segregative institutions, requiring some units to stake their claims without consideration for other units, only come about when strictly required by law. This is a qualitatively different approach than the one taken in the earlier two cases. Most importantly, it's the simultaneous presence of formal ('Coord_Units') and informal ('Early_Coord') institutions that draws the line here.

\section{Analysis 1: Integrative institutions}

Local administrations characterized by integrative institutions are expected to provide the coordination needed to adapt to climate change by adjusting available coordination structures. An operational distinction between integrative and segregative institutions has been provided. Both vulnerability to climate change and the adjustment of prior structures will be operationalized herewith through the descriptors 'Need2Adapt_Projected' and 'Fine_Tuning'.

These theoretical expectations will be confirmed if the set of those local administrations featuring integrative institutions and perceiving the need to adapt to climate change is a subset of those local administrations adjusting prior institutions. The two sets are confronted in Figure 5b. One can see that, if the need for adaptation is perceived, integrative institutions are a sufficient condition for adaptation processes - provided such processes are understood as the adjustment of existing coordination structures ('Fine_Tuning'). The sufficiency claim is technically solid (consistency score: 0.778 ) and explains a large portion of the compliant cases (coverage score: 0.636 ). Yet it is worthwhile to search the cases for an illustration of the mechanism highlighted by Figure $5 \mathrm{~b}$. 
Projecting the need to adapt to climate impacts ('Need2Adapt_Projected') and featuring both formal and informal coordination structures ('Integrative_Institutions'), LocAdm_09 is again a good term of reference. Interviews highlight the cross-cutting nature of climate adaptation and hence the need to proceed in a coordinated fashion. Interestingly, interviewees report how this became clear during past pilot projects, which have shown a broad range of affected units within the administration. They do question, though, whether these and similar processes are actually new, or whether they are better seen in the frame of the natural flexibility of administrative operations ('Fine_Tuning').

\section{Analysis 2: Segregative institutions}

This conceptualization grants a key role to uncertainty as a mechanism leading to more integration (via adaptation processes) by raising segregation costs. Segregative institutions have an ambiguous relationship with uncertainty, calling for a closer look. Figure $5 \mathrm{c}$ confronts segregative institutions with the need to adapt. As expected, no clear set relation exists between the two sets.

The absence of a clear link between segregative institutions and perceived climate vulnerability makes it worthwhile to restrict the analysis to those eight local administrations featuring segregative institutions. The role of uncertainty is captured by 'Vulnerability_ACTUAL', a set encompassing those local administrations that already 'feel' climate impact, ruling out uncertainty. This is confronted with 'Need2Adapt_Projected' in Figure 5d.

The figure shows that the two sets overlap to about $80 \%$ (consistency and coverage scores for both sufficiency and necessity: 0.8 ). Leaving technicalities aside, both sufficiency and necessity claims are contradicted by just one case, which can be tolerated. For the purposes of this analysis, the empirical evidence is compatible with a claim that local administrations characterized by segregative institutions perceive the need to adapt if and only if they already 'feel' climate impacts.

The link between vulnerability and the need to adapt is not surprising and reflects the well-known reactive character of adaptation found elsewhere in the literature. More interesting here is to characterize such a link in the context of segregative institutions. This is best done with reference to LocAdm_12. Interviews report that, when impacts are felt, problem owners understandably react and plan accordingly. More importantly, though, they also stress the need to coordinate adaptation action in order to avoid parallel work - a need for increased integration.

Addressing increased integration, Figure 5e tackles the question whether, once uncertainty is ruled out ('Vulnerability_ACTUAL') and the need to adapt is perceived ('Need2Adapt_Projected'), segregative institutions actually lead to adaptation processes ('Ongoing_Process'). This is confirmed: with the exception of just one case (LocAdm_17), featuring 'Vulnerability_ACTUAL' and 'Need2Adapt_Projected' constitutes a necessary and sufficient condition for 
'Ongoing_Process' (consistency for necessity: 1; coverage: 0.75 ; consistency for sufficiency: 0.75 ; coverage: 1 ). This is best illustrated with reference to the case of LocAdm_11.

LocAdm_11 currently experiences drought problems in its green spaces, even in spite of very recent heavy rain events ('Vulnerability_ACTUAL'). Adaptation is at the stage of planning and preparation rather than actual measures ('Need2Adapt_Projected'). In that context, some interviewees feel confident about the possibility of coordinating action through the available institutions. Other stress, instead, that adaptation is simply too integrative a task for the usual tools and procedures. As a matter of fact, several units are experimenting with new forms of coordination ('Ongoing_Process'), suggesting, as expected, a move towards integrative institutions.

\section{Discussion}

\section{Summary of the findings}

The analyses above have provided the following results. First, local administrations do differ in terms of segregative versus integrative institutions (Analysis 0). Second, this difference has a bearing on the way they approach adaptation. Under integrative institutions, local administrations tackle adaptation by adjusting available coordination structures so as to address climate change (Analysis 1). Under segregative institutions, local administrations implement adaptation processes and thus increase the amount of integration between their functional units. However, uncertainty induced by climate change is per se not sufficient to shift their segregation costs curve: for that to happen, the actual experience of climate impacts is necessary (Analysis 2). That said, caution is due while generalizing from these results: the limitations and potential pitfalls are discussed below.

\section{Limits of materials and methods}

A first limitation of the present work relates to the geographical scope of the empirical materials. The German study area is large enough to provide variation in socio-economic and cultural terms, as it includes both large and small local administrations, both rich and poor communities, both densely and sparsely populated areas. Yet it does not exhaust the variability available within a large federal country such as Germany, let alone Europe or the global north. Concerns about the context-specificity of these results should not be overemphasized, though: contrary to much adaptation and institutional research based on single case studies, the present work delivers a comparative analysis that is actually below average in terms of context-specificity.

A more serious case selection issue concerns the missing cases. The 'universe' at stake is the set of all climate-sensitive local administrations in the two chosen study areas, encompassing 25 cases. Yet only 19 could be approached. There is 
no reason to assume that particular biases are being introduced by these missing six cases. Yet it cannot be excluded that some of the analyses would come to different conclusions if a few cases were added to the dataset. This is particularly relevant for Analysis 2, which addresses only a subset of the available cases and would be therefore affected the most if additional non-compliant cases were added.

Further considerations are due with reference to the data collection and coding process. A single author performed the coding of the individual case studies, raising reliability questions. However, the case studies were based on structured interviews designed and carried out by the same author who later drafted the case studies and coded them, ensuring a much deeper knowledge of the cases than what multiple authors could have gathered from the case study texts alone.

Caution is also due with reference to the use of crisp rather than fuzzy sets. The available evidence was originally expressed using fuzzy sets, capturing the degree of ambiguity ('fuzziness') surrounding the membership of each case in each set. Such ambiguity was not considered here. The conversion from fuzzy to crisp sets is not problematic. More important is that repeating the analyses above with fuzzy sets doesn't lead to the same results. This is expected: taking ambiguity into account requires more nuanced analyses than performed here.

\section{Limits of the conceptualization}

Having highlighted the limits of materials and methods, the discussion can now focus on the paper's conceptualization. Three elements of it appear worth discussing: the link between climate uncertainty and ecological interdependencies; the effect of uncertainty upon segregation costs; and the way administrations react to such segregation costs as conceptualized herewith.

First, understanding climate change in terms of increased uncertainty over biophysical interdependencies will admittedly sound outlandish at first. Specifically, climate uncertainty pertains different future states of the environment, not its connectivity. Yet interdependencies are known at best within boundaries, and whether climate change will stay within those boundaries is indeed uncertain, effectively leading to uncertain biophysical interdependencies that may, no doubt, require substantial readjustments of resource management practices.

Second, it is worth discussing whether increasingly uncertain interdependencies actually lead to increased segregation costs. Key is the increased likelihood of 'hurting' resource users, together with the increased need to ensure the functionality of any particular resource by restricting the use of other ones. The reader shall be reminded here that segregation costs are understood strictly with reference to the action situation of the administrative unit: therefore they do not encompass the 'external costs' experienced by the resource users. Instead, they express the effort administrative units have to put into responding to users who bear such (hypothetical) external costs. 
This implies a mechanism in which resource users perceive losses, are able to attribute them to the actions of the local administration, and are able to act against the responsible unit (possibly also indirectly, via political hierarchies and/or the actions of other administrative units involved). This represents a rather long causality chain, which would be worthwhile studying on its own account. While generally plausible, such a long causality chain may indeed present non-linear and counterintuitive characteristics, potentially invalidating the mechanism postulated herewith.

In spite of all the above, it is important to stress that the mechanism at stake needs only to have cognitive, not ontological validity. The conceptualization focuses on the action situation administrative units find themselves in. It is therefore sufficient that officials realistically expect and/or anticipate reactions from the resource users. That is certainly not far-fetched. Also, if the mechanism at stake has a cognitive rather than ontological nature, counterintuitive properties are effectively ruled out: it would be rather unrealistic for administration officials to structure their expectations towards the general public by the means of elaborated algorithms.

\section{Implications for the literature}

Let us now review the implications of our findings for the literature. Starting with institutional economics, an important lesson relates to the reactive way in which institutional change takes place. In the absence of prior integrative institutions, interdependencies (and the cost of neglecting them) must be certain for new institutions to come about, transitioning from segregative to integrative institutions. This resonates well with those contributions stressing the link between shared mental models and institutions (Denzau and North, 1994) and the challenges it poses to institutional change (Bromley, 2006).

Given that uncertainty is a pervasive phenomenon when dealing with environmental resources (Vatn, 2005), this implies that many institutions are likely to be less integrative than they actually should be. For those institutional arrangements, segregation costs could be traded off against the costs of increased integration with a win. Indeed, contributions calling for inclusiveness, participation and coordination are rather frequent in the literature on environmental issues (including climate adaptation). The above could provide an institutional economic explanation for such calls.

Concerning the literature on climate adaptation, this paper's findings call first of all for a more nuanced take on the role and potential of local administrations to prepare for climate change. Scholars have not yet achieved consensus over the adaptive capacity of local administrations, particularly concerning coordination and resources (Amundsen et al., 2010; Keskitalo and Kulyasova, 2009). This paper shows that, concerning coordination, local administrations vary. Anchoring the discussion in terms of the appropriate level at which to tackle climate adaptation may therefore be misleading. 
Furthermore, administrations vary qualitatively in the way they address climate adaptation. Integrative institutions lead to the incremental adjustment of available coordination structures. Segregative institutions, on the other hand, hamper the development of integrative institutions as long as uncertainty is present. This implies that calls for increased coordination won't do. Under integrative institutions, further calls for integration equal to preaching the converted. Under segregative institutions, the focus is misplaced: administrations will increase integration, but only once they see the need to.

Amundsen et al. (2010) find that the implementation of adaptation requires a history of extreme weather events, which local governments allegedly know best. Yet this paper suggests a different story: a history (or even a present) of extreme weather events is necessary so as to overcome uncertainty, which otherwise prevents local administrations from organizing for adaptation and coordinated action. The empirical data confirm this. In these respects, experience with climate impacts has seemingly little to do with knowing where and how exactly they will hit. It has more to do with acknowledging that they will hit. The argument that local administrations know best can therefore be questioned.

Uncertainty seems to prevent local administrations characterized by segregative institutions to move towards integrative ones. Taking this circumstance at face value would imply that renewed efforts should be put into removing uncertainty from climate projections. This however, may never happen: uncertainty may be a constitutive part of the systems that climate sciences describe, not therefore being amenable to removal through ever more precise modelling efforts. In these regards, there is scope for joint efforts between institutional and the climate adaptation scholars to understand the institutional nature of uncertainty as a barrier to adaptation and explore ways to increase integration in spite of it.

\section{Conclusions}

Increasingly compelling climate reports, extreme weather events and the general failure of international politics to credibly curb greenhouse gases have provided an incredible momentum to climate adaptation. Many national governments have put climate adaptation on their agenda, yet the burden of preparing for a future of increased climate uncertainty rests mostly on the work and judgement of local administrations. Academia is divided on the very capacity of local administrations to live up to such a challenge.

Based on transaction costs economics, this paper has provided an institutional analysis of a specific question concerning how local administrations adapt: whether or not their different functional branches are expected to respond to climate change in a coordinated fashion. Questions of collective action have been present in the adaptation literature for many years now. Institutional economics analyses of those incentives for or against collective action for local adaptation 
haven't yet come about, however. There's thus a research gap to be filled, starting with the present contribution.

Focusing on the distinction between integrative and segregative institutions, this paper has provided a comparative analysis of 19 climate-sensitive local administrations in Germany. Using set-theoretical methods, the analysis shows that variation in the way local administrations structure their internal coordination determines the way they approach climate adaptation. Those administrations featuring integrative institutions will adjust prior coordination structures to accommodate the new issue. Those featuring segregative institutions will instead equip themselves with integrative institutions for climate adaptation, provided however that they already 'feel' climate change. They won't do this if climate impacts are uncertain.

\section{Acknowledgements}

This research was funded by the German Ministry for Education and Research within the econCCadapt project (grant number FKZ 01LA1137B). We wish to express our gratitude towards two anonymous reviewers for their precious comments on earlier drafts of this manuscript.

\section{Supplementary material}

To view supplementary material for this article, please visit https://doi.org/ $10.1017 /$ S1744137417000418

\section{References}

Adger, W. N. (2003): Social capital, collective action, and adaptation to climate change. In Economic Geography 79(4), pp. 387-404.

Amundsen, H., F. Berglund and H. Westkog (2010): Overcoming barriers to climate change adaptation - a question of multilevel governance? In Environment and Planning C: Government and Policy 28(2), pp. 276-89.

Baldwin, C. Y. (2007): Where do transactions come from? Modularity, transactions, and the boundaries of firms. In Industrial and Corporate Change 17(1), pp. 155-95.

Birner, R. and H. Wittmer (2004): On the 'efficient boundaries of the state': the contribution of transaction-costs economics to the analysis of decentralization and devolution in natural resource management. In Environment and Planning C: Government and Policy 22(5), pp. 667-85.

Bromley, D. W. (2006): Sufficient Reason: Volitional Pragmatism and the Meaning of Economic Institutions. Princeton: Princeton University Press.

Buchanan, J. M. and G. Tullock (1962): The Calculus of Consent: Logical Foundations of Constitutional Democracy. Ann Arbor: University of Michigan Press.

Coase, R. (1960): The problem of social cost. In Journal of Law and Economics, 3, pp. 1-44.

Denzau, A. T. and D. C. North (1994): Shared mental models: ideologies and institutions. In Kyklos 47(1), pp. 3-31. 
Dodman, D. and D. Satterthwaite (2008): Institutional capacity, climate change adaptation and the urban poor. In IDS Bulletin 39(4), pp. 67-74.

Few, R., K. Brown and E. Tompkins (2011): Public participation and climate change adaptation: avoiding the illusion of inclusion. In Climate Policy 7(1), 46-59.

Gruehn, D., S. Greiving, S. Rannow, M. Fleischhauer, B. C. Meyer, W. Loibl, J. Züger, M. Köstl, C. Diller and F. Dosch (2010): Klimawandel als Handlungsfeld der Raumordnung: Ergebnisse der Vorstudie zu den Modelvorhaben 'Raumentwicklungsstrategien zum Klimawandel'. Bundesministerium für Verkehr, Bau und Stadtentwicklung, Berlin.

Hagedorn, K. (2008): Integrative and Segregative Institutions: a Dichotomy for Nature-related Institutional Analysis. In C. Schäfer, C. Rupschus and U. J. Nagel (eds) Proceedings of the Second Green Week Scientific Conference. Marggraf. Weikersheim, pp. 26-38.

Hagedorn, K. (2015): Can the concept of integrative and segregative institutions contribute to the framing of institutions of sustainability? In Sustainability $7(1)$, pp. 584-611.

Hagedorn, K., K. Arzt and U. Peters (2002): Institutional arrangement for environmental cooperatives: a conceptual framework. In K. Hagedorn (ed.) Environmental Co-operation and Institutional Change. Cheltenham, UK: Edward Elgar, pp. 3-25.

Hinkel, J., S. Bisaro, T. E. Downing, M. E. Hofmann, K. Lonsdale, D. Mcevoy and J. D. Tàbara (2010): Learning to adapt: re-framing climate change adaptation. In M. Holme and H. Neufeldt (eds) Making Climate Change Work for Us: European Perspectives on Adaptation and Mitigation Strategies. Cambridge, UK: Cambridge University Press, pp. 113-34.

Keskitalo, E. C. H. and A. A. Kulyasova (2009): The role of governance in community adaptation to climate change. In Polar Research 28(1), pp. 60-70.

Langlois, R. N. (2002): Modularity in technology and organization. In Journal of Economic Behavior \& Organization 49(1), pp. 19-37.

Langlois, R. N. (2006): The secret life of mundane transaction costs. In Organization Studies 27(9), pp. 1389-410.

Marshall, G. R. (2013): Transaction costs, collective action and adaptation in managing complex social-ecological systems. In Ecological Economics 88, pp. 185-94.

McCann, L. (2013): Transaction costs and environmental policy design. In Ecological Economics 88, pp. 253-62.

McCann, L., B. Colby, K. W. Easter, A. Kasterine and K. V. Kuperan (2005): Transaction cost measurement for evaluating environmental policies. In Ecological Economics 52(4) (1 March), pp. 527-42.

Næss, L. O., G. Bang, S. Eriksen and J. Vevatne (2005): Institutional adaptation to climate change: flood responses at the municipal level in Norway. In Global Environmental Change 15(2), 125-38.

Pahl-Wostl, C. (2009): A conceptual framework for analysing adaptive capacity and multilevel learning processes in resource governance regimes. In Global Environmental Change 19(3), pp. 354-65.

Quaranta, M. and C. Q. Schneider (2013): How to ... use software for set-theoretic analysis: online appendix to C. Q. Schneider and C. Wagemann (2012): Set-Theoretic Methods for the Social Sciences - A Guide to Qualitative Comparative Analysis. Cambridge, UK: Cambridge University Press.

Ragin, C. C. (2006): Set relations in social research: evaluating their consistency and coverage. In Political Analysis 14(3), pp. 291-310. 
Roggero, M. (2015): Adapting institutions: exploring climate adaptation through institutional economics and set relations. In Ecological Economics 118, pp. 114-22.

Roggero, M. and O. Fritsch (2010): Mind the costs: rescaling and multi-level environmental governance in Venice Lagoon. In Environmental Management 46(1), pp. 17-28.

Schneider, C. Q. and C. Wagemann (2012): Set-Theoretic Methods for the Social Sciences A Guide to Qualitative Comparative Analysis. Cambridge, UK: Cambridge University Press.

Simon, H. A. (1962): The architecture of complexity. In Proceedings of the American Philosophical Society 106(6), pp. 467-82.

Thiel, A., C. Schleyer and T. Plieninger (2012): Wolves are mobile, while fruit trees are not! How characteristics of resources and supranational regulatory frameworks shape the provision of biodiversity and ecosystem services in Germany. In Environmental Policy and Governance 22(3), pp. 189-204.

Thiel, A., C. Schleyer, J. Hinkel, M. Schlüter, K. Hagedorn, A. Bisaro, I. Bobojonov and A. Hamidov (2016): Transferring Williamson's discriminating alignment to the analysis of environmental governance of social-ecological interdependence. In Ecological Economics 128, pp. 159-68.

Vatn, A. (2005): Institutions and the Environment. Cheltenham, UK: Edward Elgar.

Williamson, O. E. (1985): The Economic Institutions of Capitalism: Firms, Markets, Relational Contracting. New York: Free Press.

Williamson, O. E. (1998): Transaction cost economics: how it works and where it is headed. In De Economist 146(1), pp. 23-58. 